\title{
Multilayer Traffic Engineering Performance in Overlay Networks
}

Bart Puype, Didier Colle. Mario Pickavet, Piet Demeester

Department of Information Technology (INTEC), Ghent University - IBBT - IMEC

Gaston Crommenlaan 8 bus 201.9050 Gent, Belgium, bart.puype@intec.ugent.be

Abstract Multilayer Traffic Engineering uses fast switching optical networks to modify or up/downgrade upper ay: - Iogical topologies according to changing traffic patterns. The multiayer overlay model limits information exange between layers and thereby impacts overall performance.

Introduction

Nultayer traffic engineering (MTE) uses cross-layer techniques in order to not only perform traffic routing, but aiso change the logical topology seen by the traffic dynamically, building on some form of bandwidth provided by lower network layers.

in the case of IP-over-Optical networks, bandwidth between IP routers is provided by optical connections, or lightpaths. These lightpaths have bit rates that can still be handled in the electrical domain, for example, 10 Gbit/s, as opposed to the total Tbit/s bandwidth available in an optical fiber.

Because of this large discrepancy in bandwidth granularity, the decision of which lightpaths to install has a large impact on the upper layer IP network throughput performance or blocking rate, and therefore also on secondary QoS metrics such as delay, packet loss etc. Fast switching optical networks however allow to set up and tear down lightpaths dynamically, so one does no need to install a single logical IP topology. Instead, the logical topology can be adapted according to the offered IP traffic.

The possibility to install multi-hop lightpaths lowers load (and therefore cost) of IP routers. Although optical switching can be quite fast, adding or removing an IP link (forwarding adjacency) in the logical topology will require some time while the IP layer routing protocol stabilizes around the new topology. Alternatively, one can install extra (or remove) parallel lightpaths along already existing ones, which upgrades (or downgrades) IP link bandwidth in a way that is transparent to the IP layer routing protocol. However this method may be less cptimal from an optical layer perspective.

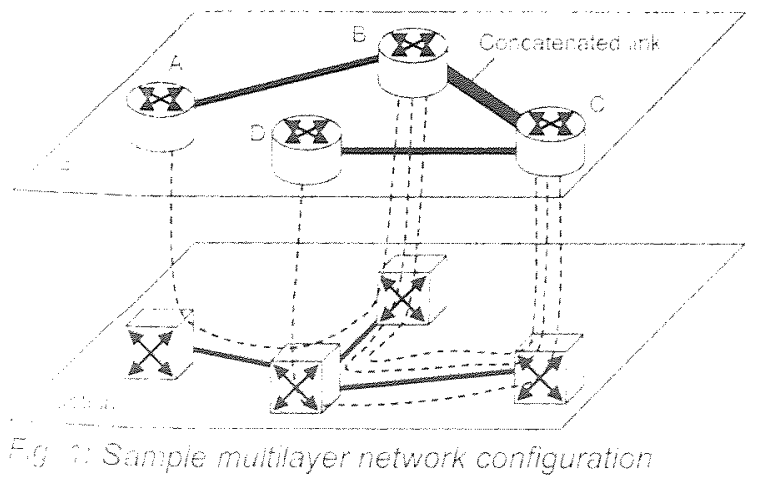

The figure shows a sample logical topology configuration for a multilayer network. Here the physical layer is a star topology, anticipating most traffic to terminate in node D. However, actual traffic patterns may differ from the originally envisioned ones; moreover patterns may change or oscillate over time. E.g. the set of heavy traffic nodes can change radically from day to nighttime. In this case, traffic appears concentrated in nodes $\mathrm{B}$ and $\mathrm{C}$ and therefore two parallel lightpaths have been installed between those routers (using for example virtual concatenation).

\section{Multilayer network models}

The introduction of optical switching technology creates several issues which did not traditionally arise in IP (electrical) networks. First of all, bandwidth granularity of lightpaths is very large, compared to the packet granularity of IP networks. This leads to suboptimal filling of circuit-switched lightpaths. Optical packet switched networks (and derivatives, e.g. burst switching), are still several years away, especially for very large networks on a national or continental scale. A second issue is the fact that wavelength translating capabilities are typically not (or only very sparsely) available in optical switches. This means that an endto-end optical connection typically remains on the same wavelength throughout the optical fiber hops. This leads to sub-optimal filling of the wavelengths on the fibers.

One view of multilayer networks is to consider an integrated model [1][2]. In this case, routing and logical topology configuration calculations are performed on a single integrated view (graph) of the IP-over-Optical network. This method typically allows for the most optimal solution. However. optical layer characteristics may lead to complications. For example, the wavelength constraint tends to be modeled by separating the optical layer in separate per-wavelength parallel graphs. Furthermore. protocol issues and economic constraints (e.g. inter-layer conficentiality wil in many cases reduce the practical vability of this approach

A second vew then is called the overlay model. In this case, the layers of the mutlayer neworks are considered separated. With no or a very limited amount of information exchange between layer control planes. This means that calculations also 
need to take into account this limited availability of information. From a practical point of view, this model is more feasible, especially when incrementally upgrading existing non-integrated infrastructure. This paper examines how this information exchange can be used to tune network performance.

\section{Multilayer traffic engineering and optical metrics} The MTE strategy used in this publication depends on information available in the IP layer through monitoring (IP flow and link bandwidths) and exchange (from the optical layer).

A worst case scenario from a resource usage perspective is to establish a full mesh for the IP layer logical topology. Although IP router load is minimal in this case, lightpath link filling is very low and the number of required optical interfaces is very high. The MTE strategy performs both IP layer routing and logical topology mesh reduction (i.e., optimization) through a single cost function which depends on IP link costs. The strategy considers one traffic flow (e.g., forwarded in an MPLS LSP) per IP router pair.

$A$ higher cost is assigned to IP links with a load below a low load threshold, in order to stimulate the mesh size reduction.

Optical information is multiplied into the IP layer cost function as an optical metric that assigns a linearly rising cost for longer (in hops) lightpaths. Several optical metrics and also the case with no optical metric were considered. A single hop lightpath has an equal cost in all of these cases. [3] describes this MTE strategy in more detail, and also examines impact of the optical metric on resource usage for the case where no parallel lightpaths can be established - however, that scenario would lead to very dense meshing for high traffic volumes.

\section{Impact on performance}

In this paper, we show the impact of the optical metrics on blocking rates. For this, we offer Poisson traffic connections to the IP layer, and have the MTE strategy groom these low bandwidth connections into higher bandwidth lightpaths. Blocking rates are determined vs. the average traffic volume between node pairs, measured in lightpath bandwidths. For the optical layer, a first-fit fixed-alternate RWA strategy was chosen.

The linear optical metrics were chosen such that a one hop lightpath has a cost of 1 for each metric. The metrics can then be characterized by their intercept on the cost-axis $C$, which is a constant fixed-cost for setting up lightpaths (i.e., the cost for a lightpath with 'zero' hops). For $\mathrm{C}=1$, cost is independent on lightpath length, for $\mathrm{C}<1$, cost becomes increasingly higher for longer lightpaths.

Lower C will promote setting up shorter lightpaths, which for the IP layer means a sparser mesh, with IP links consisting of multiple parallel lightpaths (point-topoint grooming). C close to 1 will promote end-to-end grooming instead. The influence of this parameter $\mathrm{C}$ on blocking rates is shown on fig. 3 .

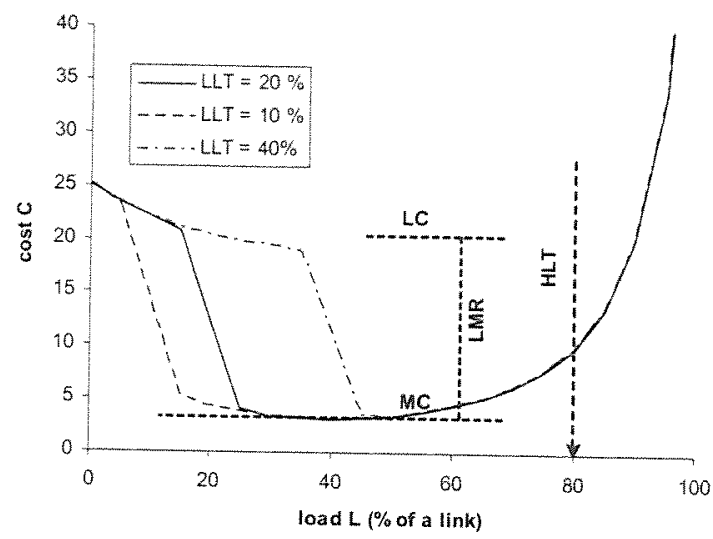

Fig. 2: IP layer cost function

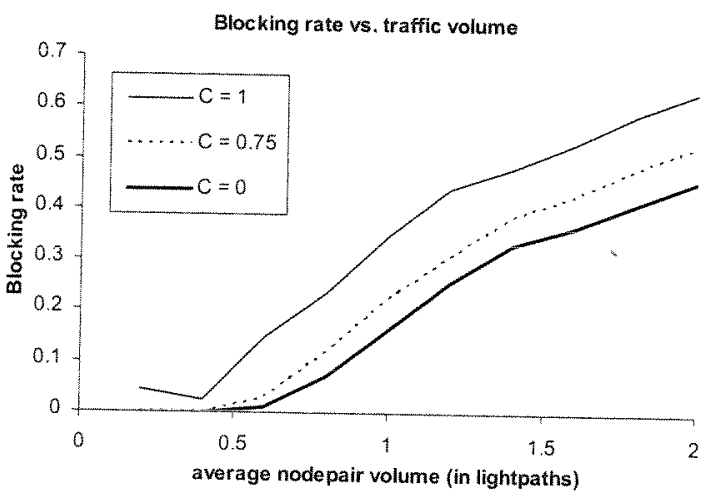

Fig. 3: Blocking rates vs. traffic volume

\section{Conclusion}

Promoting point-to-point grooming will shift resource optimization towards the optical layer, and has a significant impact on blocking rates. However, it also leads to higher transit traffic volumes in IP routers. This means a compromise has to be found between optical and IP layer optimization, by setting the optical metric parameter $C$.

\section{References}

1 Murali Kodialam et al., "Integrated dynamic IP and wavelength routing in IP over WDM networks", in Proc. IEEE INFOCOM 2001, pp.358-366;

2 T. Cinkler et al.: Faimess Issues of Routing with Grooming and Shared Protection, in Proc. ONDM 2004 pp. 665-68;

3 Bart Puype et al., "Optical cost metrics in Multlaver Traffic Engineering for IP-over-Optical neworks", in Proc. ICTON 2004, vol. 1, pp. 75-80. 


\section{ECOC 2006}

\section{2n: European Comierenoe}

\section{on Optoal communterton}

24 - 28 September 2006, Cannes, France
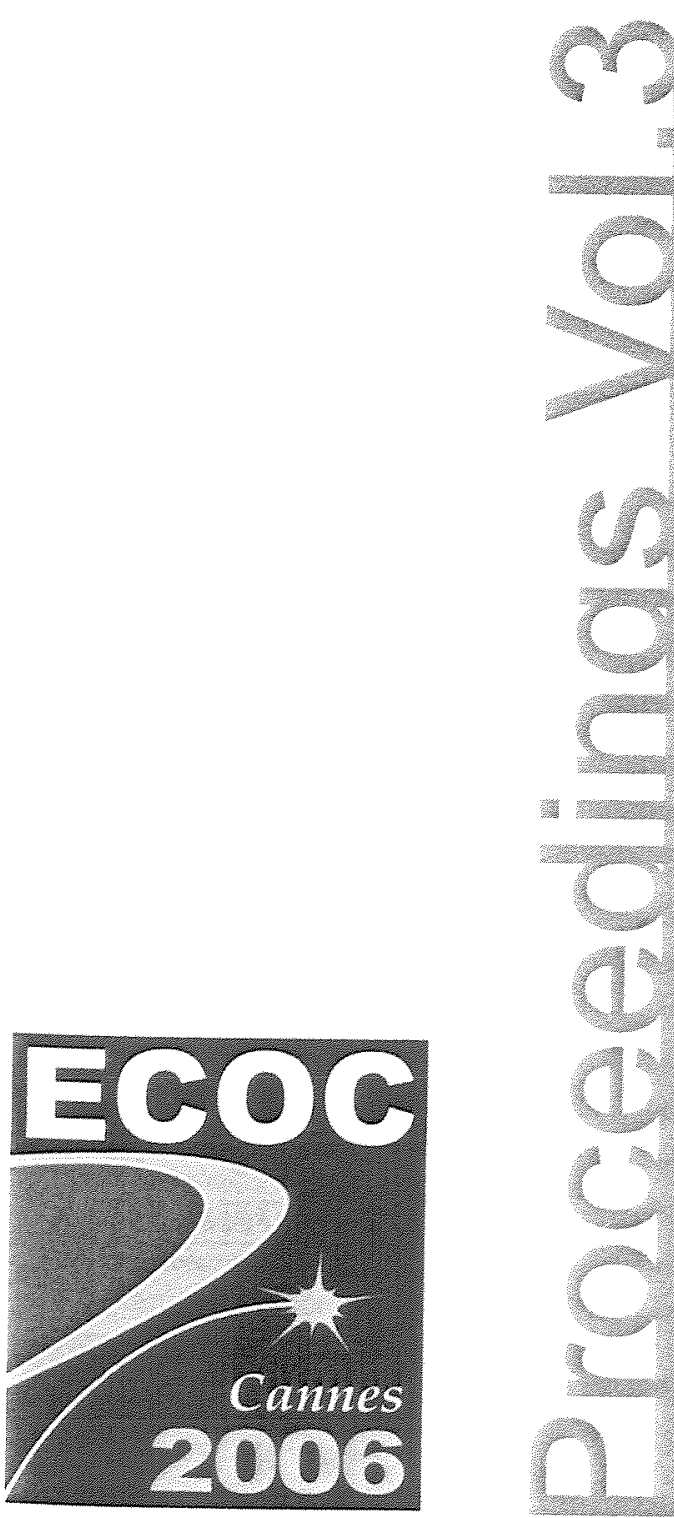

\section{Wednesday, 27 September 2006}

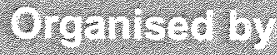

The SEE (Sociéte de lElectricite, de I'Electronique et des Technologies de I'nformation et de la Communication)

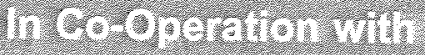

IEEELEOS (Laser and Electro-Optics

经 Society)

OSA (Optical Society of America) SFO (Societe Francaise d'Optique) EPS (European Physical Sociely) VDE Verband des Elektrotechnik, Elektronik und Informationstechnik) EUREL (Convention of National Socielies of Electrical Encineers Europe) 